\title{
Ideas Estocásticas
}

Fundamentales en el

Currículo Colombiano

Dicleny Castro Carvajal

y Antonio Moreno Verdejo

\begin{abstract}
Resumen
Los lineamientos curriculares y estándares básicos de matemáticas de Colombia, a través del Ministerio de Educación, consideran la enseñanza de la Estadística y de la Probabilidad desde la educación básica primaria, evolucionando de manera conceptual a través de la básica y media. Se analiza en este estudio la presencia de las ideas estocásticas destacadas por Shaughnessy (2019): distribuciones, la centralización, la variabilidad y la inferencia, como las cuatro ideas estocásticas fundamentales, a lo largo de toda la enseñanza obligatoria. Empleando como metodología el análisis de contenido, se logra identificar las ideas estocásticas presentes en los lineamientos curriculares de cada nivel de educación básica y media. Se encontró un énfasis en conceptos asociados a las grandes ideas sobre distribuciones estadísticas e inferencia informal. Sin embargo, estos conceptos por sí solos no son suficientes, porque requieren establecer relaciones entre estos y las actividades, procesos y contextos, que estimulen el desarrollo del razonamiento estocástico desde la educación básica primaria.
\end{abstract}

Palabras clave: Análisis curricular, Análisis de contenido, Sentido estocástico, Enseñanza de la Estadística. 


\title{
Stochastic Ideas \\ Fundamental in the \\ Colombian Curriculum
}

\begin{abstract}
The curricular guidelines and basic standards of mathematics in Colombia, through the Ministry of Education, consider the teaching of statistics and probability from primary basic education, evolving conceptually through secondary and middle school. The presence of the stochastic ideas highlighted by Shaughnessy (2019) is analyzed in this study: distributions, centralization, variability and inference, as the four fundamental stochastic ideas, throughout all compulsory education. Using the content analysis methodology, it is possible to identify the stochastic ideas present in the curricular guidelines of each level of basic and secondary education. An emphasis was found on concepts associated with big ideas about statistical distributions and informal inference. However, when appearing as concepts, no relationships are established between these and the activities, processes and contexts that stimulate the development of stochastic reasoning from primary basic education.
\end{abstract}

Keywords: Curricular analysis, Content analysis, Stochastic sense, Teaching statistics.

Nombre del trabajo: Ideas Estocásticas Fundamentales en el Currículo Colombiano

Nombre y apellido autor 1: Dicleny Castro Carvajal. Profesora de la Facultad de Ciencias de la Educación. Universidad del Tolima, Colombia.

Dirección profesional: Facultad de Ciencias de la Educación. Barrio Santa Helena, parte alta, calle 42-1 02. Ibagué Tolima.

Lugar de trabajo: Universidad del Tolima (Ibagué, Tolima).

Teléfono de contacto: 3184874091

Dirección electrónica: dcastroc@ut.edu.co
Nombre y apellido autor 2: Antonio Moreno Verdejo. Profesor del Departamento de Didáctica de la Matemática. Universidad de Granada, España. Dirección profesional: Facultad de Ciencias de la Educación. Campus Universitario Cartuja s/n, 18011. Granada.

Teléfono de contacto: 637233684

Dirección electrónica: amverdejo@ugr.es 


\section{Introducción}

Los lineamientos curriculares y estándares básicos de matemáticas del Ministerio de Educación Nacional, señalan la importancia de contribuir a los propósitos de formación de la educación para el desarrollo y el progreso de los colombianos; en ese sentido, el currículo debe ser un orientador de tales acciones que hagan posible la participación efectiva de los ciudadanos en la sociedad de la información y como afirma Niño (1998):

Iniciar un cambio profundo hacia nuevas realidades en donde las «utopías» y la imaginación de nuevos modelos de sociedad estimulen entre nosotros un hombre nuevo con una actitud mental nueva, consciente de que no hay realidades por imitar sino futuros por construir (p. 2).

Aunque la Educación Estadística viene avanzando desde hace tres décadas, se considera importante analizar cómo se incluye en el currículo de educación primaria, secundaria y media, de tal forma que sean coherentes los propósitos que se mencionan en los documentos curriculares internacionales como la guía para la evaluación y la instrucción en Educación Estadística (GAISE,2005), el Consejo Nacional de Profesores de Matemáticas (NCTM, 2000) y el Programa Internacional de Evaluación de Estudiantes (PISA, 2009) (por sus siglas en inglés) (Batanero, Arteaga y Gea, 2012) y nacionales como los Lineamientos Curriculares de Matemáticas, (1998); Estándares Básicos de Competencias en Matemáticas, (2006), del Ministerio de Educación Nacional (MEN), entre otras herramientas que retroalimentan al Sistema Educativo como la Prueba Saber (denominadas así desde el 2009) o los Derechos Básicos de Aprendizaje (2016), para identificar debilidades y fortalezas, sobre todo en la implementación del currículo; y se espera que la comunidad de investigadores y la formación docente contribuyan al logro de la articulación de sus objetivos, reflejados en las competencias y aprendizajes útiles para la vida de todo ciudadano. Desde esta perspectiva se presentarán los propósitos de la formación estadística en el currículo escolar para evidenciar las ideas fundamentales de la Estocástica más destacadas en los cinco niveles o conjuntos de grados comprendidos entre los 6 y 17 años de edad, para el caso del currículo colombiano.

Los lineamientos curriculares de matemáticas del Ministerio de Educación Nacional (MEN, 1998) sugieren la enseñanza por competencias, como la comunicación, el razonamiento y la resolución de problemas, a través de pensamientos y sistemas; sin embargo, no son tan explícitos los propósitos de su enseñanza. De esta manera, se busca exponer en este artículo las ideas fundamentales de la Estocástica, presentes en el currículo colombiano, entendida esta como el conjunto de ideas en relación con la estadística, la Probabilidad y la combinatoria que propuso Heitele en 1975:

Heitele, ofreció un conjunto de ideas fundamentales para la enseñanza de la Estocástica que incluían la Probabilidad, el espacio de la muestra, la adición y las reglas del producto para la Probabilidad, la independencia y la Probabilidad compuesta/condicional, la equidistribución, la combinatoria, las variables aleatorias y las distribuciones de Probabilidad, la simulación, el muestreo, y la Ley de los Grandes Números. Se seleccionaron como fundamentales porque, entre otras cosas, (a) son poderosas ya que cada una ayuda a situar la Probabilidad como una teoría matemática; (b) pueden enseñarse en diferentes niveles del currículo, desde la escuela primaria hasta la universidad, y los estudiantes pueden progresar en la formalización y la completitud; y (c) aparecen en la mayoría de las situaciones aleatorias (Burril y Biehler, 2011, p. 60).

Con lo anterior, el estudio de lo estocástico, amplía el horizonte de posibilidades para comprender la variabilidad, medirla y representarla, y en el sentido de Costa y Nacarato (2011): 
Lo estocástico se convierte en una herramienta que facilita y enriquece la educación para la sociedad actual, proporcionando una mayor agilidad en el uso y combinación de métodos y técnicas para resolver problemas cotidianos y profesionales en las más diversas áreas; además de permitir a cualquier persona diferentes lecturas e interpretaciones de la realidad que le rodea. Esto probablemente asegura la posibilidad de análisis y el desarrollo del razonamiento necesario e importante en la escuela primaria, mejorando la formación crítica de los estudiantes. Incluso hoy en día hay una falta de investigación sobre el tema de lo estocástico. Tal vez porque no es, por razones históricas, un tema muy tratado en las aulas de la escuela básica, como parte del currículo de Matemáticas; o tal vez porque no tiene la atención merecida dentro de los cursos de licenciatura: se forma un ciclo donde cada vez se valora menos la Estadística y todas sus herramientas (p. 370).

Investigaciones demuestran que los problemas de la enseñanza de lo estocástico, están asociados a los siguientes aspectos planteados por Shaughnessy (1992):

- El papel de la Probabilidad y la Estadística en el currículo.

- Relaciones entre investigación y educación.

- La preparación de los profesores de matemáticas.

- La forma en que se lleva a cabo el aprendizaje (p. 466).

En este artículo vamos a centrar la atención solamente sobre el papel de la Probabilidad y la estadística en el currículo, en relación con las ideas fundamentales de la Estocástica, presentes en el currículo de matemáticas. Describiendo el conjunto de ideas fundamentales en Estadística que deberían ser enseñadas en la escuela de matemáticas y que todo estudiante debería conocer al salir de la escuela básica y media. «Para Heyman, las ideas fundamentales en matemáticas deberían hacer comprensible para los estudiantes la relación entre la cultura matemática y la no matemática» (citado en Salcedo, 2019, p. 1). Es de anotar aquí, que las ideas fundamentales se están entendiendo de manera análoga a los objetivos de la educación general que contribuye al desarrollo personal, asimismo, es equivalente a lo que recomiendan los estándares básicos de competencias en matemáticas de Colombia (MEN, 2006) sobre los fines generales de la educación, que argumentan que más allá de formar a los estudiantes en el razonamiento lógico y en los conocimientos matemáticos, hay una prioridad actualmente por la necesidad de una educación básica de calidad para todos los ciudadanos, el valor social ampliado de la formación matemática y el papel de las matemáticas en la consolidación de los valores democráticos. En esta misma dirección, los estándares básicos de competencias en matemáticas (MEN, 2006) justifican el porqué de la Educación Matemática, entendida esta desde los cinco pensamientos y sistemas, incluido aquí el pensamiento aleatorio y sistema de datos, afirmando que:

La Educación Matemática debe responder a nuevas demandas globales y nacionales, como las relacionadas con una educación para todos, la atención a la diversidad y a la interculturalidad la formación de ciudadanos con las competencias necesarias para el ejercicio de sus derechos y deberes democráticos (p. 46).

Asimismo, se justifica por el reconocimiento de tres factores, a saber:

El primero, obedece al ideal de ofrecer a toda la población del país una educación básica masiva con equidad y calidad, es decir, formar en matemáticas a todo tipo de alumnos. Por ello, se hace necesario comenzar por la identificación del conocimiento matemático informal de los estudiantes en relación con las actividades prácticas de su entorno (p. 47).

El segundo factor incorpora nuevas finalidades sociales a los propósitos de la formación matemática, las cuales se argumentan con las siguientes razones: 
La primera alude al carácter utilitario ampliado del conocimiento matemático. La segunda razón alude al conocimiento matemático imprescindible y necesario en todo ciudadano para desempeñarse en forma activa y crítica en su vida social y política y para interpretar la información necesaria en la toma de decisiones (p. 47).

El tercer factor, busca contribuir desde la Educación Matemática a la formación en los valores democráticos. Esto implica reconocer que hay distintos tipos de pensamiento lógico y matemático que se utilizan para tomar decisiones informadas, para proporcionar justificaciones razonables o refutar las aparentes y falaces y para ejercer la ciudadanía crítica (p. 48).

Desde estas perspectivas será considerada la relación de la Educación Matemática con los fines de la educación. Comprendiendo adicionalmente y sin olvidar que, así como las matemáticas surgieron de una construcción humana a partir de necesidades de la humanidad, así también, esta creación contribuye a resolver problemas reales y contextuales, y mejore la sociedad en la que vivimos. Investigadores de la Educación Matemática (Bruner, 1997; Lave, 2001; Bishop, 2000; Heymann, 1996; Mora, 2009; Schweiger, 1992; Tietze, Klika y Wolpers, 1997) han considerado como ideas fundamentales de la Educación Matemática, las siguientes:

1) Idea de número, 2) idea de la medida, 3) idea de la dependencia funcional, 4) idea de la Probabilidad, 5) idea de la estructuración espacial, 6) idea del algoritmo, 7) idea de la modelación matemática, 8) idea de la comunicación intra y extra-matemática, 9) idea de la resolución de problemas intra y extra-matemáticos, y 10) idea de la matemática productiva y comunitaria. (Citados por Mora, 2010, p. 24).

Al respecto, Mora (2010) afirma lo siguiente:

Estas diez ideas básicas de la Educación Matemática pudieran ser consideradas como la relación más estre- cha y directa entre las matemáticas y la complejidad cultural de cada pueblo a lo largo y ancho del mundo. El verdadero significado e importancia de las matemáticas debe ilustrarse mediante el tratamiento de situaciones problemáticas completamente diferentes al mundo de las matemáticas "convencionales», normalmente practicadas en la cotidianidad de los momentos sociomatemáticos al interior de los procesos educativos formales, correspondientes a los requerimientos de la sociedad capitalista (pp. 24-25).

Los propósitos de la educación expresados a través de competencias, nos remite al objetivo y definición de currículo para la Educación Matemática, en el sentido que lo afirma Rico y Lupiañez (2008):

Las matemáticas forman parte de la tradición cultural de nuestra sociedad, contribuyen de manera singular a la formación de las personas, al desarrollo de sus facultades, al cultivo de su carácter. Igualmente, las matemáticas proporcionan herramientas para la actividad laboral y el ejercicio profesional y forman para la ciudadanía (p. 33).

A la siguiente definición de currículo que presenta Rico y Lupiáñez (2008), agregaría la pregunta ¿A quiénes se enseña?, en el sentido de reconocer el contexto, sus particularidades y sus necesidades:

El currículo de la educación obligatoria es un plan de formación que en su diseño y desarrollo, tiene que dar respuesta concreta a las siguientes cuestiones: ¿Qué formación? ¿Con cuál conocimiento?, la formación, ¿para qué?, ¿Qué aprendizaje se persigue?, ¿Cómo llevar a cabo la formación? ¿Cuál fue la formación? ¿Cuáles resultados se obtuvieron? Esto es, un plan de formación, que responda a las necesidades individuales y demandas sociales del momento (p. 34).

Lo anterior, tiene dos propósitos en este artículo: el primero, es reconocer la contribución que hace la formación matemática a los fines de la educación, en 
un sentido universal; $y$ el segundo propósito es tener un punto de referencia más específico de las ideas fundamentales de la Educación Matemática respecto a las ideas fundamentales de la Estadística, presentes en el pensamiento aleatorio y sistema de datos, y será en este último en el que nos vamos a detener. En Colombia, Vasco (1998) propone el «concepto de sistema» como eje organizador de los lineamientos curriculares de matemáticas, clasificándolos en cinco sistemas: numéricos, geométricos, de medidas, de datos y algebraicos y analíticos; luego en MEN (1998) se retoma el concepto de «sistema» y cada uno de ellos se enriquece con un pensamiento: numérico, espacial, métrico, aleatorio y variacional; finalmente en MEN (2006), permanece con los cinco sistemas y sus respectivos pensamientos, pero ahora en el contexto de «Estándares básicos de competencias».

Estos sistemas, pensamientos y Estándares básicos de competencias se organizan desarrollados en cinco grupos de grados $1^{\circ}$ a $3^{\circ}$ (edades entre 6 a 8 años), $4^{\circ}$ a $5^{\circ}$ (edades entre 9 y 10 años), $6^{\circ}$ a $7^{\circ}$ (entre 11 y 12 años), 8 a 9을 (edades entre 13 y 14 años) y $10^{\circ}$ a $11^{\circ}$ (edades entre 15 y 16 años), graduando el nivel de complejidad en competencias de comunicación, razonamiento y resolución de problemas a la vez que identifica procesos generales como formular y resolver problemas; modelar procesos y fenómenos de la realidad; comunicar; razonar y formular, comparar y ejercitar procedimientos y algoritmos. Estos a su vez, mediados por diferentes contextos, ambientes y situaciones de aprendizaje significativo y comprensivo de las matemáticas (MEN, 2006).

La investigación en educación estadística ha tomado un importante interés en la comunidad de profesores de matemáticas en Colombia, por la necesidad de implementar el pensamiento aleatorio y sistema de datos recomendado en los Estándares Básicos de Competencias en Matemáticas (MEN, 2006). Se requiere que los profesores con las nuevas demandas de la sociedad actual, logre con la incorporación de la estadística en el currículo escolar, formar ciudadanos competentes. En este sentido Rodríguez-Alveal (2017) expresa la relación de la persona con su entorno:

En este contexto la formación de los escolares juega un rol fundamental debido a la gran cantidad de información económica, educativa, social, cultural y política que se presenta mediante representaciones gráficas o indicadores numéricos. Ejemplo de ello son las cifras que hacen referencia a promedios y porcentajes, como asi también los datos en coherencia con el tipo de muestreo cuya finalidad es reforzar la credibilidad de la información resumida (p. 1460).

Lo anterior permite participar de manera efectiva en la construcción de una sociedad estadísticamente culta, formación ciudadana que va a permitir aportar a la construcción de una sociedad crítica basada en información real y contextual.

Por la responsabilidad que tiene el profesor con el desarrollo del currículo, deberá promover no solo el desarrollo de competencias, sino también, la cultura estadística en la educación básica y media, en el sentido que Wallman (1993) la define: «la actitud crítica hacia la información estadística y la valoración de la estadística como una habilidad para la toma de decisiones» (citado por Contreras y Molina, 2019, p.35). En la misma línea, Batanero (2013) concibe el sentido estadístico como la unión de tres componentes:

- Comprensión de las ideas estadísticas fundamentales a un nivel adecuado. Ideas que aparecen en la mayoría de las situaciones en que hay que aplicar la Estadística.

- Competencia de análisis de datos.

- El razonamiento estadístico, que incluye los elementos anteriormente descritos.

En este artículo, se muestra cómo están explicitas o implícitas ideas fundamentales de la Estadística y de la Probabilidad en los Estándares Básicos de Competencias en Matemáticas (MEN, 2006), como guía en la educación básica y media vocacional del 
diseño y regulador curricular de las Matemáticas en Colombia. Además, porque el pensamiento aleatorio y sistema de datos, está incluido en la enseñanza obligatoria de la Educación Matemática, a través de los lineamientos curriculares, situación que no ha permitido diferenciarlas como asignaturas y por ende, tampoco se dedica tiempo a identificar la naturaleza y sus propios objetos de estudio. Shaughnessy (2019) enfatiza que mientras la Matemática está basada en el razonamiento determinista anclado en la axiomática, las estadísticas implican la toma de decisiones que percibimos que es más probable que sean verdaderas en base a los datos que se generan bajo condiciones de incertidumbre. En definitiva, como señala Moreno (2017), podemos concretar las razones que avalan la enseñanza y aprendizaje de la Estadística y la Probabilidad en las siguientes:

- Alfabetización estadística. Adquirir la capacidad de lectura de gráficos y datos estadísticos que se han incorporado como parte del lenguaje de los sistemas de comunicación.

- Competencia estadística. Ser capaz de integrar el problema real y el problema estadístico para plantear y/o resolver problemas estocásticos.

- Competencia para tomar decisiones. El estudiante formado en análisis de datos desarrolla un mejor espíritu crítico frente a estadísticas manipuladas o sesgadas con objeto de influir en el individuo o la opinión pública.

- Curriculares. El currículo debe mostrar también los aspectos no deterministas de las ciencias.

\section{Ideas fundamentales de la Estocástica}

Las contribuciones realizadas entre profesores de estadística y profesores de matemáticas e investigadores a través de las conferencias internacionales como la ICOTS, (Conferencia Internacional sobre Estadísticas de la Enseñanza), organizaciones como la ASA (Asociación Estadística Americana),y el NCTM
(Consejo Nacional de Profesores de Matemáticas) y los proyectos financiados por USEI y la IASE (Asociación Internacional de Educación Estadística). A nivel nacional, el Encuentro Colombiano de Educación Estocástica (ECEE), ha realizado su tercera edición (2018), el Encuentro Internacional de la Enseñanza de la Probabilidad y Estadística (EIEPE), en México, el Encuentro de Probabilidades y estadística en la escuela en Portugal, o el Encuentro de Didáctica de la Estadística, la Probabilidad y el Análisis de Datos (EDEPA) en Costa Rica y el Grupo de Investigación en Educación Estadística, liderado por Batanero, de la Universidad de Granada con una trayectoria de 30 años de investigación por la Educación Estadística y recientemente el III Congreso Internacional Virtual de Educación Estadística (2019), ayudando con ello a vincular progresivamente a investigadores interesados en la educación estocástica (Batanero, 2019). Asimismo, se estudia a la educación estocástica como otro componente de la investigación de la Educación Matemática, como lo demuestran los grupos de trabajo sobre la educación estocástica en el ICME (Congreso Internacional de Educación Matemática), las conferencias del CERME (Conferencias Europeas de Educación Matemática), el RELME (Conferencia Latinoamericana de Educación Matemática) y los simposios, como el SEIEM (Simposios de la Sociedad Española de Educación Matemática) (Batanero, 2019).

Las ideas fundamentales de la Estocástica, es un tema que ha sido objeto de estudio por distintos autores como Kilpatrick, Swafford y Findell (2001), Watson (2006), Kilpatrick, Swafford y Findell (2001), Garfield y Ben-Zvi (2008), Burrill y Biehler (2011) y Batanero y Borovcnik (2016), (citados en Salcedo, 2019, p.2) entre otros. Se encuentra con especial atención en este estudio, que la mayoría de los autores han considerado a la variabilidad como idea fundamental en el razonamiento de la Estocástica (ver Tabla 1).

A continuación, se muestran en la Tabla 1, los conceptos centrales que cada autor sugiere como recomendaciones sobre las grandes ideas en edu- 
cación estocástica y cuáles de estas son recurrentes en la medida que avanza la investigación en esta área, reconociéndolas como ideas fundamentales. Posteriormente, estas ideas serán contrastadas con las competencias propuestas en el pensamiento aleatorio y sistema de datos para el currículo co- lombiano. Sin embargo, en este estudio, Shaughnessy (2019), encontró que algunas ideas estaban incluidas en otras más representativas, como las Distribuciones, la Variabilidad, la centralización y la Inferencia. Sobre estas 4 ideas Estocásticas centraremos nuestra atención.

\begin{tabular}{|c|c|c|c|c|c|c|c|c|c|}
\hline \multicolumn{10}{|c|}{ Ideas fundamentales de la Estocástica } \\
\hline $\begin{array}{l}1 . \\
\text { David } \\
\text { Moore } \\
(1990)\end{array}$ & \begin{tabular}{|l|} 
\\
Wild y \\
Pfannkuch \\
$(1999)$
\end{tabular} & $\begin{array}{l}3 . \\
\text { Kilpatrick, } \\
\text { Swafford y } \\
\text { Findell } \\
(2001)\end{array}$ & $\begin{array}{l}4 . \\
\text { Watson } \\
(2006)\end{array}$ & $\begin{array}{l}5 . \\
\text { Garfield y } \\
\text { Ben-Zvi } \\
(2008)\end{array}$ & $\begin{array}{l}6 . \\
\text { Burrill } \\
\text { y Biehler } \\
(2011)\end{array}$ & $\begin{array}{l}7 . \\
\text { Watson } \\
\text { et al. } \\
(2013)\end{array}$ & $\begin{array}{l}8 . \\
\text { Kader \& } \\
\text { Jacobbe } \\
(2013)\end{array}$ & $\begin{array}{l}9 . \\
\text { Batanero y } \\
\text { Borovcnik } \\
(2016)\end{array}$ & $\begin{array}{l}10 . \\
\text { Shaugh- } \\
\text { nessy } \\
\text { (2019) }\end{array}$ \\
\hline $\begin{array}{l}\text { - Variabi- } \\
\text { lidad }\end{array}$ & $\begin{array}{l}\text { - Variabi- } \\
\text { lidad }\end{array}$ & $\begin{array}{l}\text { - Describir } \\
\text { los datos } \\
\text { - Organizar } \\
\text { los datos } \\
\text { - Representar } \\
\text { los datos } \\
\text { - Espacio } \\
\text { muestral } \\
\text { - Probabilidad } \\
\text { de un evento } \\
\text { - Probabilidad } \\
\text { de compa- } \\
\text { raciones } \\
\text { a través de } \\
\text { espacios } \\
\text { de muestra } \\
\text { - Probabilidad } \\
\text { condicional } \\
\text { e indepen- } \\
\text { dencia }\end{array}$ & $\begin{array}{l}\text { - Colección } \\
\text { de datos } \\
\text { - Representa- } \\
\text { ción gráfica } \\
\text { - Reducción } \\
\text { de datos } \\
\text { - Variabilidad } \\
\text { - Inferencia } \\
\text { - Muestreo } \\
\text { - Probabilidad }\end{array}$ & $\begin{array}{l}\cdot \text { Datos } \\
\cdot \text { Distribución } \\
\cdot \text { - Variabilidad } \\
\cdot \text { Centro } \\
\cdot \text { Modelos } \\
\text { estadísticos } \\
\cdot \text { - Covariación } \\
\cdot \text { Inferencia } \\
\cdot \text { Aleatorie- } \\
\text { dad } \\
\cdot \text { Muestreo }\end{array}$ & $\begin{array}{l}\cdot \text { Datos } \\
\cdot \text { - Variabilidad } \\
\text { - Distribución } \\
\text { - Representa- } \\
\text { ción gráfica } \\
\text { - Asociación } \\
\text { - Modelación } \\
\text { de relación } \\
\text { entre dos } \\
\text { variables } \\
\text { - Muestreo } \\
\text { - Inferencia } \\
\text { - Modelos de } \\
\text { Probabilidad }\end{array}$ & $\begin{array}{l}\cdot \text { Variación } \\
\cdot \text { Expectativa } \\
\cdot \text { Distribu- } \\
\text { ción } \\
\cdot \text { Azar } \\
\text { - Inferencia }\end{array}$ & $\begin{array}{l}\text { - Variabilidad } \\
\text { - Distribución } \\
\text { - Asociacio- } \\
\text { nes entre } \\
\text { dos variables } \\
\text { - Muestras y } \\
\text { poblaciones }\end{array}$ & $\begin{array}{l}\text { - Análisis } \\
\text { exploratorio } \\
\text { de datos } \\
\text { - Modelación } \\
\text { de infor- } \\
\text { mación por } \\
\text { Probabilidad } \\
\text { - Exploración } \\
\text { - Modelación } \\
\text { de la asocia- } \\
\text { ción } \\
\text { - Muestreo } \\
\text { - Inferencia }\end{array}$ & $\begin{array}{l}\text { · Distribu- } \\
\text { ción } \\
\text { · Inferencia }\end{array}$ \\
\hline
\end{tabular}

Tabla 1.

Se señalaron con color gris las ideas que Shaugnessy (2019) considera, estarían incluidas en la idea de Variabilidad, con color azul las que están incluidas en las Distribuciones, con color rosa las que están incluidas en la idea de Centro y con color naranja, las ideas de Inferencia (formal e informal).

Fuente: elaboración propia. 


\section{Metodología}

Para el propósito que tiene este estudio se realizará un análisis de contenido (Krippendorff, 1990) dentro de un enfoque cualitativo; de la estructura del sistema educativo de Colombia, se presentan los estándares básicos de competencias de matemáticas (2006), en particular, serán analizados los que corresponden al Pensamiento Aleatorio y Sistema de Datos, propuestos desde el primer ciclo de básica primaria hasta la educación media, que, en nuestro caso, son los dos últimos años de bachillerato; documento que es diseñado y producido por el Ministerio de Educación Nacional en Colombia (2006). Se busca evaluar e identificar la presencia de las cuatro ideas estocásticas fundamentales estudiadas por Shaughnessy (2019), a la luz de las investigaciones que ha realizado, y que se usarán en este estudio a manera de antecedentes según la Tabla 1, frente a los contenidos del currículo colombiano.

La muestra fue intencionada y está conformada por 41 estándares de competencias del pensamiento aleatorio de la educación básica y media en Colombia, como documento curricular (MEN, 2006), que guía los planes de aula respecto a los conceptos relacionados con la educación Estocástica.
Para llevar a cabo el análisis, se han identificado los estándares que hacen referencia a las cuatro ideas estocásticas fundamentales identificadas por Shaugnessy (2019): Distribuciones, Centralización, Variabilidad e Inferencia, en los cinco niveles de la educación básica y media en Colombia. Además, se analizó su recurrencia, para ver donde se encontraban concentradas. Estas ideas estocásticas pueden tener presencia más de una vez en cada uno de los estándares de competencias, por lo tanto, para efectos del análisis se cuentan estas frecuencias de manera independiente para cada idea estocástica fundamental, señaladas por Shaughnessy. Considerando esto, se obtuvieron 85 respuestas, que se distribuyen como se muestran en las tablas 3 , 4, 5, 6 y 7, en la columna 3 respectivamente; así mismo, están cuantificadas como se presentan en las gráficas 1, 2, 3, 4, 5 .

A continuación, en la Tabla 2, se presentan los conceptos y características que permiten leer las ideas estocásticas, anteriormente mencionadas y con las que se van a identificar en el currículo colombiano. Para ello, se tendrán en cuenta las ideas de Daniel (1995). 


\begin{tabular}{|c|c|c|c|}
\hline 1. Distribuciones & $\begin{array}{l}\text { 2. Medidas } \\
\text { de centralización }\end{array}$ & $\begin{array}{l}\text { 3. Medidas } \\
\text { de Variabilidad }\end{array}$ & 4. Inferencia \\
\hline $\begin{array}{l}\text { - La Distribución de } \\
\text { frecuencias es un método } \\
\text { para organizar y resumir } \\
\text { datos. } \\
\text { - Se usa para clasificar, } \\
\text { ordenar y contar las veces } \\
\text { que se repite un dato. } \\
\text { - Permite resumir grandes } \\
\text { conjuntos de datos. } \\
\text { - Representa las cate- } \\
\text { gorías numéricas de la } \\
\text { variable junto con el } \\
\text { número de entidades } \\
\text { que se clasifican en cada } \\
\text { categoría. } \\
\text { - Se pueden presentar en } \\
\text { forma tabular o forma } \\
\text { gráfica. } \\
\text { - Realiza intervalos de } \\
\text { clase según la cantidad } \\
\text { de datos. }\end{array}$ & $\begin{array}{l}\text { - Una medida de tenden- } \\
\text { cia central es el único nú- } \\
\text { mero que indica el centro } \\
\text { de una serie de números } \\
\text { a partir de los cuales se } \\
\text { calcula. } \\
\text { - También se llaman medi- } \\
\text { das de localización. } \\
\text { - Implica concepto de } \\
\text { promedio. } \\
\text { - Aunque son varias las } \\
\text { medidas de tendencia } \\
\text { central, las más usadas } \\
\text { son la media aritmética, } \\
\text { la mediana y la moda. }\end{array}$ & $\begin{array}{l}\text { - La Variabilidad tiene } \\
\text { como sinónimo el de } \\
\text { dispersión. } \\
\text { - Medidas utilizadas para } \\
\text { ver la manera en que los } \\
\text { valores individuales se } \\
\text { desvían del «promedio». } \\
\text { - Hacen parte de estas } \\
\text { medidas de Variabilidad: } \\
\text { El rango, la varianza y la } \\
\text { desviación típica. }\end{array}$ & $\begin{array}{l}\text { Inferencia Informal: } \\
\text { Makar y Rubin (2018); } \\
\text { Zeiffler, Garfield, Del Mas } \\
\text { y Reading (2008) (citados } \\
\text { por Shaughnessy, 2019, } \\
\text { p. 12) } \\
\text { - Estimar las Probabilida- } \\
\text { des a partir de muestras } \\
\text { de datos. } \\
\text { - No implica hacer estadís- } \\
\text { tica de prueba o distribu- } \\
\text { ción de Probabilidad. } \\
\text { - Permite hacer argu- } \\
\text { mentos sobre muestras } \\
\text { observadas (de poblacio- } \\
\text { nes desconocidas). } \\
\text { - Permite tomar decisiones } \\
\text { a partir de un conjunto de } \\
\text { datos. } \\
\text { - Es útil para hacer análi- } \\
\text { sis y conclusiones a partir } \\
\text { de un conjunto de datos. } \\
\text { - Es describir los datos. }\end{array}$ \\
\hline
\end{tabular}

Tabla 2.

Ideas fundamentales de la Estocástica. Fuente: elaboración propia. 


\section{Ideas Fundamentales en el Pensamiento Aleatorio y Sistemas de Datos del Currículo Colombiano}

A continuación, se presentan los estándares de competencias de matemáticas, en particular las recomendaciones para la enseñanza del pensamiento aleatorio y sistema de datos en Colombia, para estudiantes desde la básica primaria (considerada desde los 6 años de edad) hasta la educación media (para estudiantes entre 16 y 17 años de edad). El propósito es presentar una relación del currículo de estadística y Probabilidad, al que llamaremos en este artículo, de la «educación estocástica», con la clasificación de ideas fundamentales de la
Estocástica que presenta (Shaugnessy, 2019), como cuatro grandes ideas estadísticas en la educación de nuestros alumnos. Afirma que progresivamente han sido consideradas las siguientes ideas fundamentales: la centralización, la variabilidad, las distribuciones y la inferencia informal. Esta clasificación de Shaugnessy sobre las ideas fundamentales de la Estocástica, será de interés en este trabajo para compararlas y ver en qué estándares de competencias del pensamiento aleatorio y sistema de datos, están concentradas.

\begin{tabular}{|c|c|}
\hline Estándar Básico de Competencia & $\begin{array}{l}\text { Ideas Fundamentales } \\
\text { de la Estocástica }\end{array}$ \\
\hline $\begin{array}{l}\text { Clasifico y organizo datos de acuerdo a cualidades y atributos y los presento } \\
\text { en tablas. }\end{array}$ & Distribuciones \\
\hline Interpreto cualitativamente datos referidos a situaciones del entorno escolar. & $\begin{array}{l}\text { Distribuciones } \\
\text { Inferencia Informal }\end{array}$ \\
\hline Describo situaciones o eventos a partir de un conjunto de datos. & Inferencia Informal \\
\hline $\begin{array}{l}\text { Represento datos relativos a mi entorno usando objetos concretos, pictogra- } \\
\text { mas y diagramas de barras. }\end{array}$ & Distribuciones \\
\hline Identifico regularidades y tendencias en un conjunto de datos. & $\begin{array}{l}\text { Distribuciones } \\
\text { Variabilidad } \\
\text { Centralización }\end{array}$ \\
\hline $\begin{array}{l}\text { Explico - desde mi experiencia- la posibilidad o imposibilidad de ocurrencia } \\
\text { de eventos cotidianos. }\end{array}$ & $\begin{array}{l}\text { Distribuciones } \\
\text { Inferencia Informal }\end{array}$ \\
\hline Predigo si la posibilidad de ocurrencia de un evento es mayor que la de otro. & Inferencia Informal \\
\hline $\begin{array}{l}\text { Resuelvo y formulo preguntas que requieran para su solución coleccionar y } \\
\text { analizar datos del entorno próximo. }\end{array}$ & $\begin{array}{l}\text { Distribuciones } \\
\text { Variabilidad } \\
\text { Inferencia Informal }\end{array}$ \\
\hline
\end{tabular}

Tabla 3.

Pensamiento aleatorio y Sistemas de datos. Básica Primaria, primer nivel, grados $1^{\circ} a 3^{\circ}$. Fuente: MEN (2006). 
La Tabla 3 muestra la vinculación entre las competencias del pensamiento aleatorio y sistema de datos, recomendados en los lineamientos y estándares curriculares (MEN, 2006) para grados $1^{\circ}, 2^{\circ}$ y $3^{\circ}$ de básica primaria y las cuatro ideas fundamentales de la Estocástica, haciendo especial énfasis en las distribuciones y la inferencia informal. Según los propósitos de la investigación en educación estadística, estos marcos deben ayudar al estudiante a reconocer la centralización, la forma y la variabilidad de un conjunto de datos, que en el mejor de los casos deben ser recolectados por ellos mismos y que le sirvan de evidencia para la toma de decisiones. Makar y Rubin (2018) consideran que, en esta etapa, «los estudiantes de primaria logran hacer inferencia informal basados en los datos que han recogido de una pregunta estadística cuando ellos mismos la han planteado, así mismo, que el conocimiento contextual desempeñan un papel en el análisis y la inferencia» (citado por Shaughnessy, 2019, p. 12).

\begin{tabular}{|l|l|}
\hline Estándar Básico de Competencia & $\begin{array}{l}\text { Ideas Fundamentales } \\
\text { de la Estocástica }\end{array}$ \\
\hline $\begin{array}{l}\text { Represento datos usando tablas y gráficas (pictogramas, gráficas de barras, } \\
\text { diagramas de líneas, diagramas circulares). }\end{array}$ & Distribuciones \\
\hline Comparo diferentes representaciones del mismo conjunto de datos. & Distribuciones \\
\hline $\begin{array}{l}\text { Interpreto información presentada en tablas y gráficas. (pictogramas, gráficas } \\
\text { de barras, diagramas de líneas, diagramas circulares). }\end{array}$ & $\begin{array}{l}\text { Distribuciones } \\
\text { Inferencia Informal }\end{array}$ \\
\hline $\begin{array}{l}\text { Conjeturo y pongo a prueba predicciones acerca de la posibilidad de ocurrencia } \\
\text { de eventos. }\end{array}$ & $\begin{array}{l}\text { Distribuciones } \\
\text { Inferencia Informal }\end{array}$ \\
\hline $\begin{array}{l}\text { Describo la manera como parecen distribuirse los distintos datos de un conjun- } \\
\text { to de ellos y la comparo con la manera como se distribuyen en otros conjuntos } \\
\text { de datos. }\end{array}$ & $\begin{array}{l}\text { Distribuciones } \\
\text { Centralización } \\
\text { Variabilidad }\end{array}$ \\
\hline $\begin{array}{l}\text { Uso e interpreto la media (o promedio) y la mediana y comparo lo que indican. } \\
\text { observaciones, consultas o experimentos. }\end{array}$ & \begin{tabular}{l} 
Centralización \\
\hline $\begin{array}{l}\text { Resuelvo yormulo problemas a partir de un conjunto de datos provenientes de } \\
\text { Distribuciones } \\
\text { Inferencia Informal }\end{array}$
\end{tabular} \\
\hline
\end{tabular}

\section{Tabla 4.}

Pensamiento aleatorio y Sistemas de datos. Básica Primaria, segundo nivel, grados $4^{\circ} a 5^{\circ}$. Fuente: MEN (2006).

En la Tabla 4, para el segundo nivel de educación básica primaria, predomina la enseñanza de las distribuciones, sugiriendo con este marco, que los estudiantes agudicen la observación de los datos, se hagan preguntas y puedan conjeturar. De esta manera se refuerza lo visto y desarrollado en el primer nivel de la educación básica.
En la Tabla 5 se presentan los estándares de competencias para básica secundaria y se avanza en otros marcos; además de las distribuciones y la inferencia, aparecen según el análisis de contenido, la centralización y la variabilidad. De esta manera, se hace necesario identificar, describir, interpretar y resolver problemas con contenido estadístico, en la búsqueda de argumentos que ayuden en la etapa del análisis. 


\begin{tabular}{|c|c|}
\hline Estándar Básico de Competencia & $\begin{array}{l}\text { Ideas Fundamentales } \\
\text { de la Estocástica }\end{array}$ \\
\hline $\begin{array}{l}\text { Comparo e interpreto datos provenientes de diversas fuentes (prensa, revistas, } \\
\text { televisión, experimentos, consultas, entrevistas). }\end{array}$ & $\begin{array}{l}\text { Distribuciones } \\
\text { Variabilidad } \\
\text { Inferencia informal }\end{array}$ \\
\hline Reconozco la relación entre un conjunto de datos y su representación. & Distribuciones \\
\hline $\begin{array}{l}\text { Interpreto, produzco y comparo representaciones gráficas adecuadas para pre- } \\
\text { sentar diversos tipos de datos. (Diagramas de barras, diagramas circulares.) }\end{array}$ & $\begin{array}{l}\text { Distribuciones } \\
\text { Variabilidad } \\
\text { Inferencia informal }\end{array}$ \\
\hline $\begin{array}{l}\text { Uso medidas de tendencia central (media, mediana, moda) para interpretar } \\
\text { comportamiento de un conjunto de datos. }\end{array}$ & $\begin{array}{l}\text { Centralización } \\
\text { Inferencia informal }\end{array}$ \\
\hline $\begin{array}{l}\text { Uso modelos (diagramas de árbol, por ejemplo) para discutir y predecir posibili- } \\
\text { dad de ocurrencia de un evento. }\end{array}$ & $\begin{array}{l}\text { Variabilidad } \\
\text { Inferencia informal }\end{array}$ \\
\hline $\begin{array}{l}\text { Conjeturo acerca del resultado de un experimento aleatorio usando proporciona- } \\
\text { lidad y nociones básicas de Probabilidad. }\end{array}$ & $\begin{array}{l}\text { Centralización } \\
\text { Inferencia informal }\end{array}$ \\
\hline $\begin{array}{l}\text { Resuelvo y formulo problemas a partir de un conjunto de datos presentados en } \\
\text { tablas, diagramas de barras, diagramas circulares. }\end{array}$ & $\begin{array}{l}\text { Distribuciones } \\
\text { Inferencia informal }\end{array}$ \\
\hline Predigo y justifico razonamientos y conclusiones usando información estadística. & $\begin{array}{l}\text { Distribuciones } \\
\text { Inferencia informal }\end{array}$ \\
\hline
\end{tabular}

Tabla 5.

Pensamiento aleatorio y Sistemas de datos. Básica Secundaria, tercer nivel, grados $6^{\circ} a 7^{\circ}$. Fuente: MEN (2006).

En la Tabla 6 se presentan los estándares para el desarrollo del pensamiento aleatorio en estudiantes del cuarto nivel de educación básica, y se observa que predominan las ideas de distribuciones, variabilidad e inferencia informal sin embargo, la idea de centralización no es tan recurrente, al parecer, empieza a quedar implícita en la idea de las distribuciones. Se espera que los estudiantes en esta etapa avancen hacia el dominio de conceptos y procedimientos necesarios para recoger, estudiar, resumir y representar sistemas de datos estadísticos.
La Tabla 7 muestra los estándares básicos de competencias del pensamiento aleatorio para el último nivel, con el que culmina la educación media, y se evidencian las cuatro ideas fundamentales de la Estocástica, aunque sigue predominando la enseñanza de las distribuciones, solo que en este nivel predominan las distribuciones de Probabilidad y se presenta de manera más evidente la idea de la centralización con respecto a los niveles anteriores de la educación. 


\begin{tabular}{|c|c|}
\hline Estándar Básico de Competencia & $\begin{array}{l}\text { Ideas Fundamentales } \\
\text { de la Estocástica }\end{array}$ \\
\hline $\begin{array}{l}\text { Reconozco cómo diferentes maneras de presentación de información pueden originar } \\
\text { distintas interpretaciones. }\end{array}$ & $\begin{array}{l}\text { Distribuciones } \\
\text { Variabilidad } \\
\text { Inferencia informal }\end{array}$ \\
\hline $\begin{array}{l}\text { Interpreto analítica y críticamente información estadística proveniente de diversas } \\
\text { fuentes (prensa, revistas, televisión, experimentos, consultas, entrevistas. }\end{array}$ & $\begin{array}{l}\text { Distribuciones } \\
\text { Variabilidad } \\
\text { Inferencia informal }\end{array}$ \\
\hline $\begin{array}{l}\text { Interpreto y utilizo conceptos de media, mediana y moda y explicito sus diferencias } \\
\text { en distribuciones de distinta dispersión y asimetría. }\end{array}$ & $\begin{array}{l}\text { Distribuciones } \\
\text { Centralización } \\
\text { Variabilidad }\end{array}$ \\
\hline $\begin{array}{l}\text { Selecciono y uso algunos métodos estadísticos adecuados al tipo de problema, de } \\
\text { información y al nivel de la escala en la que esta se representa (nominal, ordinal, de } \\
\text { intervalo o de razón). }\end{array}$ & $\begin{array}{l}\text { Distribuciones } \\
\text { Variabilidad }\end{array}$ \\
\hline $\begin{array}{l}\text { Comparo resultados de experimentos aleatorios con los resultados previstos por un } \\
\text { modelo matemático probabilístico. }\end{array}$ & $\begin{array}{l}\text { Distribuciones } \\
\text { Variabilidad } \\
\text { Inferencia formal }\end{array}$ \\
\hline $\begin{array}{l}\text { Resuelvo y formulo problemas seleccionando información relevante en conjuntos de } \\
\text { datos provenientes de fuentes diversas (prensa, revistas, televisión, experimentos, } \\
\text { consultas, entrevistas). }\end{array}$ & $\begin{array}{l}\text { Distribuciones } \\
\text { Variabilidad } \\
\text { Inferencia informal }\end{array}$ \\
\hline Reconozco tendencias que se presentan en conjuntos de variables relacionadas. & $\begin{array}{l}\text { Distribuciones } \\
\text { Centralización } \\
\text { Variabilidad }\end{array}$ \\
\hline $\begin{array}{l}\text { Calculo Probabilidad de eventos simples usando métodos diversos (listados, diagra- } \\
\text { mas de árbol, técnicas de conteo). }\end{array}$ & Distribuciones \\
\hline Uso conceptos básicos de Probabilidad (espacio muestral, evento, independencia, etc.). & Distribuciones \\
\hline
\end{tabular}

Tabla 6.

Pensamiento aleatorio y Sistemas de datos. Básica Secundaria, cuarto nivel, grados $8^{\circ}$ a $9^{\circ}$. Fuente: MEN (2006). 


\begin{tabular}{|c|c|}
\hline Estándar Básico de Competencia & $\begin{array}{l}\text { Ideas Fundamentales } \\
\text { de la Estocástica }\end{array}$ \\
\hline $\begin{array}{l}\text { Interpreto y comparo resultados de estudios con información estadística provenientes } \\
\text { de medios de comunicación. }\end{array}$ & $\begin{array}{l}\text { Distribuciones } \\
\text { Variabilidad } \\
\text { Inferencia informal }\end{array}$ \\
\hline $\begin{array}{l}\text { Justifico o refuto inferencias basadas en razonamientos estadísticos a partir de resul- } \\
\text { tados de estudios publicados en los medios o diseñados en el ámbito escolar. }\end{array}$ & $\begin{array}{l}\text { Distribuciones } \\
\text { Variabilidad } \\
\text { Inferencia informal }\end{array}$ \\
\hline $\begin{array}{l}\text { Diseño experimentos aleatorios (de las Ciencias Físicas, Naturales o Sociales) para } \\
\text { estudiar un problema o pregunta. }\end{array}$ & $\begin{array}{l}\text { Variabilidad } \\
\text { Inferencia informal }\end{array}$ \\
\hline Describo tendencias que se observan en conjuntos de variables relacionadas. & $\begin{array}{l}\text { Distribuciones } \\
\text { Centralización } \\
\text { Variabilidad }\end{array}$ \\
\hline $\begin{array}{l}\text { Interpreto nociones básicas relacionadas con el manejo de información (como población, } \\
\text { muestra, variable aleatoria, distribución de frecuencias, parámetros y estadígrafos). }\end{array}$ & $\begin{array}{l}\text { Distribuciones } \\
\text { Centralización } \\
\text { Variabilidad }\end{array}$ \\
\hline $\begin{array}{l}\text { Uso comprensivamente algunas medidas de centralización, localización, dispersión y } \\
\text { correlación (percentiles, cuartiles, centralidad, distancia, rango, varianza, covarianza } \\
\text { y normalidad). }\end{array}$ & $\begin{array}{l}\text { Centralización } \\
\text { Variabilidad }\end{array}$ \\
\hline Interpreto conceptos de Probabilidad condicional e independencia de eventos. & Distribuciones \\
\hline $\begin{array}{l}\text { Resuelvo y planteo problemas usando conceptos básicos de conteo y Probabilidad (combi- } \\
\text { naciones, permutaciones, espacio muestral, muestreo aleatorio, muestreo con remplazo). }\end{array}$ & Distribuciones \\
\hline Propongo inferencias a partir del estudio de muestras probabilísticas. & $\begin{array}{l}\text { Inferencia formal } \\
\text { Distribuciones }\end{array}$ \\
\hline
\end{tabular}

\section{Tabla 7.}

Pensamiento aleatorio y Sistemas de datos. Básica Secundaria, quinto nivel, grados $10^{\circ}$ a $11^{\circ}$. Fuente: MEN (2006).

En general, estas competencias están caracterizadas por el conocimiento conceptual y procedimental, y están relacionadas con el saber qué, el saber qué hacer y el saber cómo, cuándo y por qué hacerlo. Sin embargo, estas expresiones no se presentan de manera explícita en la enseñanza y se requieren de ambientes de aprendizaje que favorezcan «una noción amplia de competencia como conjunto de conocimientos, habilidades, actitudes, comprensiones y disposiciones cognitivas, socioafectivas y psicomotoras apropiadamente relacionadas entre sí para facilitar el desempeño flexible, eficaz y con sentido de una actividad en contextos relativamente nuevos y retadores» (MEN, 2006, p. 49).

El pensamiento aleatorio y sistema de datos, sugiere que el profesor de matemáticas considere otros procesos de pensamiento distintos a los de las matemáticas, que diferencie sus objetos y sus propósitos, en suma, su naturaleza de estudio; se requiere más reflexión y más exploración sobre las ideas fundamentales de la Estocástica para que cumplan su propósito de contribuir al sentido estocástico, en una sociedad en la que abunda la información. 


\section{Análisis y Discusión de los Resultados}

A continuación, se analizan los resultados para cada uno de los niveles de la educación básica y media, en relación con la presencia de las cuatro ideas estocásticas estudiadas por Shaughnessy (2019), presentes en los estándares de competencias del currículo colombiano.

La gráfica 1 evidencia en qué proporción se encuentran las 4 ideas fundamentales de la Estocástica en los estándares de primero a tercero de básica primaria, indicando que predominan las distribuciones y la inferencia informal. Esto puede obedecer a que se considera a las distribuciones como la primera representación de los datos para poderlos describir y en consecuencia, la necesidad de hacer inferencia informal, en el sentido que se mencionó en la tabla 2, para analizar y describir un conjunto de datos. La variabilidad y la centralización están presentes en la medida que logran identificar regularidades y tendencias en un conjunto de datos y cuando colectan los datos. Es de anotar, que aquí los niños aún no usan medidas de centralización ni de dispersión. La gráfica 2 evidencia en qué proporción se encuentran las 4 ideas fundamentales de la Estocástica en los estándares de cuarto a quinto de básica primaria, indicando que continúan predominando las distribuciones y la inferencia informal, como en el nivel anterior. Sin embargo, aumenta la presencia de los estándares que relacionan el concepto de centralización, indicando por explícito la necesidad de usar e interpretar las media y la mediana, como una manera de complementar la descripción de los datos. La gráfica 3 evidencia en qué proporción se encuentran las 4 ideas fundamentales de la Estocástica en los estándares de sexto a séptimo en secundaria, mostrando un aumento significativo de la idea de inferencia informal, posiblemente porque debe aumentar su capacidad para interpretar y describir los datos, en la medida que aumentan las representaciones; continúa por explicito el uso de las medidas de centralización, y la variabilidad continúa de manera implícita en el sentido que reconoce diferencias entre individuos o poblaciones, en general, cuando compara los datos.

La gráfica 4 evidencia en qué proporción se encuentran las 4 ideas fundamentales de la Estocástica en los estándares de octavo a noveno en secundaria, mostrando un aumento en las ideas de distribuciones y variabilidad, presentes en la recolección, la comparación de datos y en la resolución de problemas. Aparece por primera vez, la inferencia formal, por el estándar que le sugiere comparar resultados de experimentos aleatorios con los resultados previstos por un modelo matemático probabilístico. La gráfica 5 evidencia en qué proporción se encuentran las 4 ideas fundamentales de la Estocástica en los estándares de décimo a undécimo en la educación media, indicando según el análisis de contenido que continúan predominando las distribuciones y la variabilidad, como en el nivel anterior; hay un aumento en la inferencia informal y se mantiene un estándar relacionado con la inferencia formal, que sugiere proponer inferencias a partir del estudio de muestras probabilísticas. Se presenta de manera explícita la necesidad de usar comprensivamente las medidas de centralización y de dispersión y la resolución de problemas está concentrada en los conceptos de Probabilidad. 


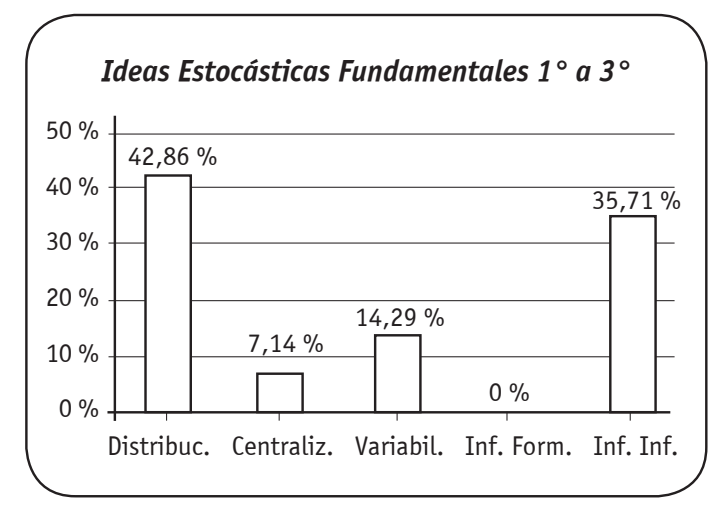

Figura 1.

Presencia de las ideas Estocásticas en los estándares del nivel 1 de básica primaria.

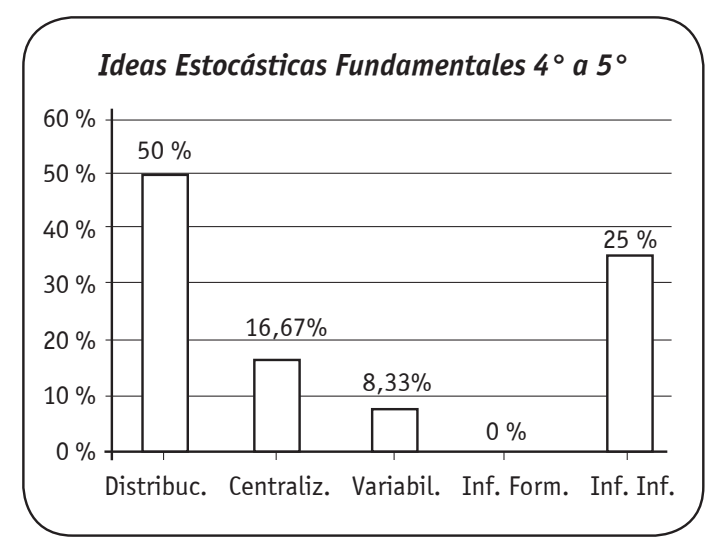

Figura 2.

Presencia de las ideas Estocásticas en los estándares del nivel 2 de básica primaria.

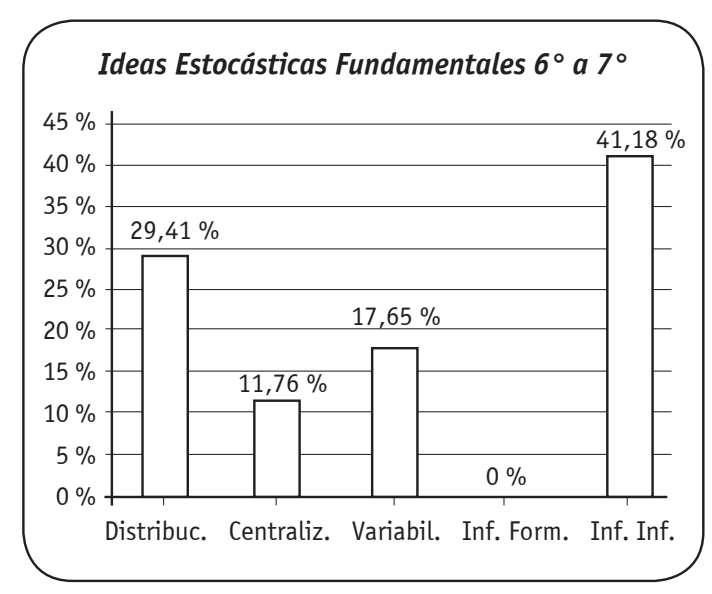

Figura 3.

Presencia de las ideas Estocásticas en los estándares del nivel 3 de básica secundaria.
Ideas Estocásticas Fundamentales $8^{\circ} a 9^{\circ}$

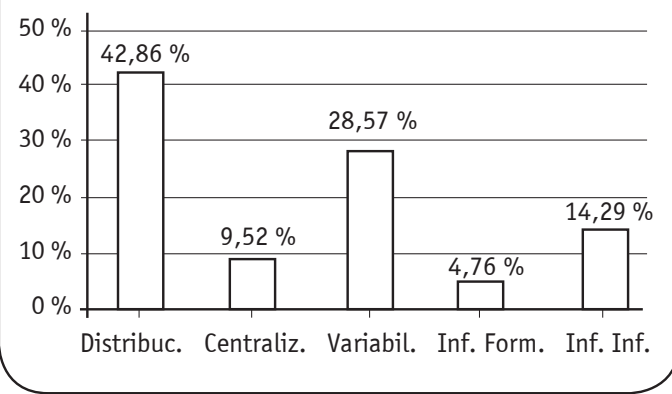

Figura 4.

Presencia de las ideas Estocásticas en los estándares del nivel 4 de básica secundaria.

Ideas Estocásticas Fundamentales $10^{\circ}$ a $11^{\circ}$

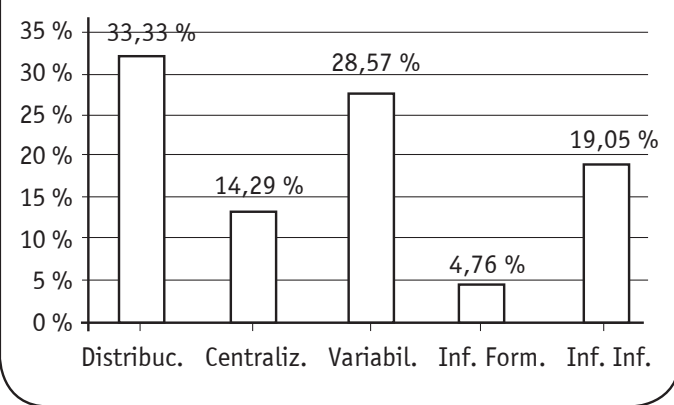

Figura 5.

Presencia de las ideas estocásticas en los estándares del nivel 5 de la educación media. 


\section{Conclusiones}

De acuerdo con los resultados obtenidos, se encontró que efectivamente las 4 ideas estocásticas fundamentales, revisadas por Shaughnessy (2019) a través de la investigación, también están presentes en el currículo colombiano, aunque de una manera muy desproporcionada. Es decir, predominan según su aporte en el currículo, de la siguiente manera: las distribuciones $(38,8 \%)$, la inferencia informal (tal como se describió en la tabla 2) $(24,71 \%)$, la variabilidad $(22,3 \%)$, la centralización (11,8\%) y la inferencia formal (como se describió en la tabla2) (2,3\%).

La estructura de los estándares básicos de competencia en matemáticas del MEN (2006), manejan una coherencia vertical y horizontal en relación con los conceptos y la gradualidad del aprendizaje, es decir, que mientras la coherencia vertical está dada por la relación de un estándar con los demás estándares del mismo pensamiento en los otros conjuntos de grados, la coherencia horizontal está dada por la relación que tiene un estándar determinado con los estándares de los demás pensamientos dentro del mismo conjunto de grados. Esta organización del currículo hace que sea más extenso el recorrido para la apropiación de los conceptos. Sin olvidar las limitaciones de tiempo, pues aún, la Estadística, no ha sido considerada como asignatura para la educación básica primaria, por ejemplo.

En lo que se refiere a los estándares del pensamiento aleatorio y sistemas de datos, se pudo analizar que las ideas conceptuales de la Estocástica (distribuciones, centralización, variabilidad e inferencia), aparecen fragmentadas, haciendo énfasis en los conceptos, más no en los procesos, situación que puede causar dificultades en el aprendizaje y en el desarrollo adecuado del razonamiento estocástico. Esta revisión ha permitido que logremos identificar que en el tratamiento de las situaciones que involucran fenómenos estocásticos (la estadística, la Probabilidad y la combinatoria) se hace necesario, la presencia de las 4 ideas fundamentales de la estocástica señaladas por Shaughnessy (2019).

Este estudio deja abierta la posibilidad para evaluar la implementación del currículo en cada uno de los niveles de educación básica y media, en relación con las cuatro ideas estocásticas fundamentales, superando los conceptos.

La presencia de las ideas fundamentales de la Estocástica en el currículo de Colombia contribuye a la formación de un ciudadano que se enfrenta con la complejidad proveniente de la incertidumbre. Tal como lo definen las competencias de matemáticas para el pensamiento aleatorio del Ministerio de Educación Nacional:

El empleo cada vez más generalizado de las tablas de datos y de las recopilaciones de información codificada llevó al desarrollo de la estadística descriptiva y al estudio de los sistemas de datos, por medio del pensamiento aleatorio llevó a la estadística inferencial y a la teoría de Probabilidades. El manejo y análisis de los sistemas de datos se volvió inseparable del pensamiento aleatorio (MEN, 2006, p.65).

«Sin embargo, en la práctica son todavía pocos los profesores que enseñan este tema y en otros casos se trata muy brevemente, 0 en forma excesivamente formalizada» (Batanero, 2000, p.6). De esta manera seguimos encontrando tensiones y limitaciones para lograr el desarrollo del razonamiento estadístico y más aún, desde la educación básica.

Para indagaciones posteriores es necesario comparar si los conceptos y procesos recomendados en el currículo de estadística, se ven reflejados y garantizan el razonamiento estadístico en actividades conectadas con las vivencias de los estudiantes, en general, se debe dar cuenta sobre su implementación. 


\section{Referencias bibliográficas}

Batanero, C. (2000). ¿Hacia dónde va la educación estadística? Blaix, 15, 2-13.

Batanero, C., Arteaga, P. y Gea, M. (2012). El Currículo de Estadística: Reflexiones desde una Perspectiva Internacional. UNO. Revista de didáctica de las matemáticas, 59, 9-17.

Batanero, C. (2013). Sentido estadístico. Componentes y desarrollo. I Jornadas Virtuales de Didáctica de la Estadística, la Probabilidad y la Combinatoria: Granada.

Batanero, C. (2019). Treinta años de investigación en educación estocástica: Reflexiones y desafíos. En J.M. Contreras, M.M. Gea, M.M. López-Martín y E. Molina-Portillo (Eds.), Actas del Tercer Congreso Internacional Virtual de Educación Estadística. Granada: CIVEEST III. Disponible en www.ugr.es/local/ fqm126/civeest.html

Burril, G. y Biehler, R. (2011). Fundamental Statistical Ideas in the School Curriculum and in Training Teachers. En C. Batanero, G. Burrill, and C. Reading (Eds.), Teaching Statistics in School Mathematics-Challenges for Teaching and Teacher Education: A Joint ICMI/IASE Study. doi: DOI 10.1007/978-94-007-1131-0_10.

Contreras, J.M. y Molina Portillo, E. (2019). Alfabetización estadística. 25 años de la evolución de un término. Números. Revista de Didáctica de las Matemáticas, 100, 35-38.

Costa, A. y Nacarato, A.M. (2011). A Estocástica na Formação do Professor de Matemática: percepções de professores e de formadores. Bolema. [en línea] Brasil: Boletim de Educação Matemática 24(39), 367386. Consultado el 26 de 0ctubre de 2019 en: https://www.redalyc.org/pdf/2912/291222099003.pdf Daniel, W. (1995). Estadística con aplicaciones a las ciencias sociales y a la educación. (traducción de Jesús Villamizar). México: McGraw-Hill.

Del-Pino, J. (2017). Sintesis de la investigación sobre variabilidad y dispersión en estadística. (Tesis de Máster) Universidad de Granada. Recuperado de: https://www.ugr.es/ ${ }^{\text {batanero/documentos/ }}$ TFMPino.pdf

Krippendorff, K. (1990). Metodología de análisis de contenido. Teoría y práctica. España: Paidós. Makar, K. \& Rubin, A. (2018). Learning about statistical inference. In: D. Ben-Zvi, K. Makar, J. Garfield (eds) International Handbook of Research in Statistics Education (pp. 261-294). Springer, Cham. Ministerio de Educación Nacional (MEN) (1998). Serie Lineamientos curriculares Matemáticas. Bogotá: Ministerio de Educación Nacional.

Ministerio de Educación Nacional (MEN) (2006). Estándares Básicos de Competencias en Lenguaje, Matemáticas, Ciencias y Ciudadanas. Bogotá, Colombia: Imprenta Nacional de Colombia.

Ministerio de Educación Nacional (MEN) (2016). Derechos Básicos de Aprendizaje. Bogotá: Panamericana Formas E Impresos S.A.

Mora, D. (2010). Formación matemática como parte de la educación integral básica (EIB) de todas las personas. Vol. III $/ \mathrm{N}^{\circ} 2$.

Moreno, A. (2017). ¿Por qué enseñamos Estadística y Probabilidad? UNO, 76, pp. 9-15.

NCTM (2000). Principles and Standards for School Mathematics. Reston, VA: National Council of Teachers of Mathematics.

Niño, J. (1998). Serie de Lineamientos Curriculares de Matemáticas. Ministerio de Educación Nacional. Bogotá. Recuperado de: https://www.mineducacion.gov.co/1621/articles-89869_archivo_pdf9.pdf Rico, L. y Lupiáñez, J. (2008). Competencias matemáticas desde una perspectiva curricular. Madrid: Alianza Editorial.

Rodríguez-Alveal, F. (2017). Alfabetización Estadística en Profesores de Distintos Niveles formativos. En Revista Educação \& Realidade, Porto Alegre, v. 42, n. 4, p. 1459-1477. Consultado el 2 de Enero de 2020. Recuperado de : http://dx.doi.org/10.1590/2175-623662610 
Salcedo, A. (2019). Las ideas fundamentales de la estadística en textos escolares de matemáticas. En J.M. Contreras, M.M. Gea, M.M. López-Martín y E. Molina-Portillo (Eds.), Actas del Tercer Congreso International Virtual de Educación Estadística. CIVEEST III. Disponible en: www.ugr.es/local/fqm126/ civeest.html.

Shaugnessy, M. (1992). Research in probability and statistics: reflections and directions. In: Grouws, D.A. (Ed.). Handbook of Research on Mathematics Teaching and Learning. USA: NCTM. p. 466.

Shaugnessy, M. (2019). The Big Ideas in the statistics education of our students: Which ones are the biggest? En XV Conferencia Interamericana de Educación Matemática-CIAEM. Medellín, Colombia.

Vasco, C. (1998). Serie de Lineamientos curriculares de matemáticas del Ministerio de Educación Nacional. Bogotá. Recuperado de: http://master2000.net/recursos/fotos/74/documentos/lineamientos $\% 20$ curriculares $\% 20$ ciencias $\% 20$ naturales.pdf 